Revista Verde de Agroecologia e Desenvolvimento Sustentável

http://www.gvaa.com.br/revista/index.php/RVADS

NOTA TÉCNICA

DOI: http://dx.doi.org/10.18378/rvads.v10i2.3096

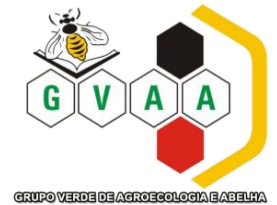

\title{
Características Agronômicas de seis Cultivares de Bananeiras tipo Prata
}

\section{Agronomic traits of six cultivars of the banana Pome type}

\author{
Tânia Santos Silva ${ }^{1}$, Sérgio Luiz Rodrigues Donato ${ }^{2}$,Vagner Alves Rodrigues Filho ${ }^{3}$, Milton Celso Padilha Júnior ${ }^{4}$, Yago $^{5}$ \\ Cândido Pereira da Silva
}

Resumo: A despeito da ampla aceitação comercial da 'Prata-Anã' é necessário o aumento do portfólio de cultivares tipo Prata para os agricultores. Objetivou-se avaliar características agronômicas de bananeiras Prata em dois ciclos de produção. Utilizouse delineamento experimental inteiramente casualizado, com seis tratamentos, 'Prata-Anã', 'Maravilha', 'BRS Platina', 'BRS Fhia-18', 'Fhia-18' e 'JV42-135', cinco repetições e quatro plantas úteis por parcela. A 'Maravilha' é a mais produtiva, com maior massa das pencas, maior massa, comprimento e diâmetro do fruto; 'JV42-135' apresenta maior porte, menos pencas e frutos; 'BRS Fhia-18' é menos vigorosa; 'BRS Platina' assemelha-se mais à genitora, 'Prata-Anã'.

Palavras-chaves: Musa spp., avaliação de genótipos, florescimento, rendimento.

\begin{abstract}
Despite the widespread commercial acceptance of 'Dwarf Pome' is required increasing portfolio of cultivars Pome type for farmers. This study aimed to evaluate agronomic traits in Pome bananas during two production cycles. A completely randomized design was adopted with six treatments, 'Dwarf Pome', 'Gold Finger', 'BRS Platina', 'BRS FHIA-18', 'FHIA-18' and 'JV42-135', five replicates in plots with four plants per plot were usedo. A 'Gold Finger' is the most yield, with greater weight of the bunches, more mass, length and fruit diameter, 'JV42-135' features larger, and less fruit bunches. 'BRS FHIA-18' is less vigorous; 'BRS Platina' resembles more the mothers, 'Dwarf Pome'.
\end{abstract}

Key words: Musa spp., genotypes evaluation, flowering, yield.

\footnotetext{
*Autor para correspondência

Recebido para publicação em 05/01/2015; aprovado em 20/05/2015.

${ }^{1}$ Acadêmicos do Curso de Agronomia, Instituto Federal Baiano, Campus Guanambi-BA, E-mail: tania_ifbaiano@hotmail.com, vagner_loiola@yahoo.com.br padilhajrmc@gmail.com yagocangbii@gmail.com, ,.

${ }^{2}$ Eng. Agro., Professor, Instituto Federal Baiano, Campus Guanambi-BA, E-mail: sergio.donato@guanambi.ifbaiano.edu.br
} 


\section{INTRODUÇÃO}

O cultivo da bananeira é bastante expressivo nos sistemas agrícolas das zonas agroecológicas dos trópicos (AZEVEDO et al., 2010), assumindo nessas regiões grande importância econômica e social (SILVA et al., 2003). No Brasil as cultivares mais difundidas são as bananas tipo prata ('Prata', 'Pacovan' e 'Prata-Anã'). No Sudoeste da Bahia (DONATO et al., 2009) e no Norte de Minas Gerais a principal variedade cultivada é a 'Prata-Anã' que apesar da boa aceitação comercial é suscetível às sigatokas e ao mal-doPanamá. Esta doença tem causado sérias restrições de cultivo em algumas propriedades, principalmente as de solo mais arenoso (RODRIGUES et al., 2011).

$\mathrm{Na}$ busca por soluções, o Programa Brasileiro de Melhoramento da Bananeira, coordenado pela Embrapa Mandioca e Fruticultura desenvolveu diversos híbridos de bananeira tipo Prata. Foram lançadas 'Maravilha' e 'BRS Fhia18', como alternativas à 'Prata-Anã' (DONATO et al., 2009) e recentemente a 'BRS Platina'. A 'Fhia-18', já em uso pelos produtores e a 'JV42-135' encontram-se em fase de avaliação em diferentes regiões brasileiras. Há também a 'PrataCatarina' ('SCS451 Catarina'), selecionada pela Epagri (LICHTEMBERG e LICHETEMBERG, 2011) e a 'PrataGorutuba' selecionada por técnicos do Norte de Minas (Rodrigues et al., 2011; Rodrigues et al., 2012).

A avaliação agronômica é fundamental para recomendação de uma cultivar. Esses estudos envolvem caracteres de rendimento, relevantes para a identificação e a seleção de indivíduos superiores (AMORIM et al., 2009), de modo a assegurar a extrapolação dos resultados e subsidiar sistemas de produção específicos. A despeito de diversas avaliações serem conduzidas com os mesmos genótipos, em ambientes diferentes e até repetidas vezes no mesmo ambiente, esse procedimento é justificável e necessário para verificação da repetibilidade e herdabilidade de algumas características e a maior influência ambiental sobre outras, o que pode conduzir à recomendação de cultivares para regiões específicas. O objetivo deste trabalho foi avaliar características agronômicas de seis cultivares de bananeiras tipo Prata em dois ciclos de produção.

\section{MATERIAL E MÉTODOS}

O trabalho foi realizado entre maio de 2010 e maio de 2012, na área experimental do Instituto Federal Baiano Campus Guanambi, coordenadas $14^{\circ} 13$ '30"S e $42^{\circ} 46^{\prime} 53^{\prime \prime} \mathrm{W}$, altitude de $545 \mathrm{~m}$, média anual de $680,00 \mathrm{~mm}$ de precipitação e temperatura média de $26^{\circ} \mathrm{C}$.

Utilizou-se o delineamento experimental inteiramente casualizado, com seis tratamentos e cinco repetições. Os tratamentos foram as cultivares de bananeira: Prata-Anã (AAB) e os híbridos tetraplóides (AAAB) Maravilha (Fhia-01), BRS Platina (PA42-44), BRS Fhia-18 e Fhia-18 (PA94-01), derivados da Prata-Anã, e JV42-135, derivado da Prata de Java. As parcelas experimentais foram constituídas por quatro plantas úteis, com bordadura externa. Os caracteres foram mensurados nos estádios de florescimento e de colheita dos cachos de cada planta útil no primeiro e segundo ciclos de produção, seguindo os procedimentos adotados por Donato et al. (2006, 2009). Altura da planta, perímetro do pseudocaule ao nível do solo, número de folhas funcionais, área foliar total (ZUCULOTO et al., 2008), índice de área foliar, massa das pencas, número de pencas e de frutos por cacho, massa, comprimento e diâmetro do fruto. Os dados obtidos foram submetidos à análise de variância e as médias comparadas pelo Teste de Tukey, a 5\% de probabilidade.

\section{RESULTADOS E DISCUSSÃO}

No primeiro ciclo de produção 'JV42-135' e 'BRS Fhia-18' foram mais alta e mais baixa, respectivamente (Tabela 1). 'Maravilha', 'Fhia-18', 'Prata-Anã' e 'BRS Platina' apresentaram porte intermediário e semelhante. No segundo ciclo 'JV42-135' manteve a maior altura, 'BRS Platina' foi similar à 'BRS Fhia-18', mas também não diferiu da 'PrataAnã', 'Fhia-18' e 'Maravilha' expressaram porte intermediário. 'Prata-Anã' e 'BRS Platina' externaram o porte semelhante nos dois ciclos de produção, fato comprovado por Donato et al. (2006, 2009), Lédo et al. (2008), Oliveira et al. (2008) e Marques et al. (2011), o que assegura verificar a manutenção do caráter da genitora na progênie.

No primeiro ciclo o perímetro do pseudocaule variou de $86,97 \mathrm{~cm}$ na 'BRS Fhia-18' a 107,21 cm para a 'Fhia-18' (Tabela 1). 'Fhia-18' foi a mais vigorosa junto com a 'Maravilha', que não diferiu da 'JV42-135'. 'Prata-Anã' e 'Platina' apresentaram vigor intermediário e a 'BRS Fhia-18' o menor vigor. No segundo ciclo 'Maravilha' foi a mais vigorosa junto com a 'Fhia-18', que foi similar à 'Prata-Anã' e à 'JV42-135'. 'BRS Platina' e 'BRS Fhia-18' foram as menos vigorosas. A detecção de maiores perímetros do pseudocaule obtida nesse estudo comparada à Donato et al. $(2006,2009)$ está relacionada ao fato do perímetro ser mensurado ao nível do solo no presente trabalho e à $30 \mathrm{~cm}$ do solo nos trabalhos revisados. Adicionalmente, diferenças na fertilidade do solo e no manejo da cultura podem ter favorecido um maior crescimento vegetativo.

A 'Prata-Anã' exibiu mais folhas funcionais no florescimento que 'Fhia-18' e 'BRS Fhia-18', e foi similar à 'Maravilha', 'BRS Platina' e 'JV42-135' (Tabela 1). No segundo ciclo, 'Prata Anã' superou as demais cultivares. O vigor da planta em bananeira é refletido por caracteres morfológicos como altura da planta, perímetro do pseudocaule e número de folhas vivas (Faria et al., 2010). Depreende-se que no geral a 'BRS Fhia-18' mostra-se menos vigorosa.

Da análise da área foliar total no primeiro ciclo de produção, nota-se que apenas a 'BRS Fhia-18' diferiu das demais, com a menor média, $9,88 \mathrm{~m}^{2}$ (Tabela 1). No segundo ciclo, 'Prata-Anã' com 18,38 $\mathrm{m}^{2}$ de AFT superou 'Fhia-18', 'BRS Platina' e 'BRS Fhia-18'. 'Maravilha' e 'JV42-135', foram similares à 'Prata-Anã' e também à 'Fhia-18', 'BRS Platina' e 'BRS Fhia-18'. Os valores de AFT seguiram a lógica do número de folhas, pois esta é proporcional à quantidade e dimensões das folhas. No local de condução do presente trabalho não há incidência sigatokas, porém a ocorrência de ventos com picos elevados de velocidade (DONATO et al., 2006, 2009) é causa destruição foliar.

Referente ao caráter índice de área foliar 'BRS Platina', 'Fhia-18', e 'Prata-Anã' se igualaram com médias intermediarias em primeiro ciclo; 'Maravilha' e 'JV42-135' expressaram os maiores índices e 'BRS Fhia-18' o menor. No segundo ciclo, a variação foi similar ao que ocorreu para AFT, uma vez que o IAF é a AFT dividida pelo espaçamento ocupado pelas plantas.

Em relação ao número de folhas avaliado por ocasião da colheita do primeiro ciclo houve uma variação de 9,70 
Tabela 1. Características agronômicas avaliadas em bananeiras tipo Prata, em dois ciclos de produção, Guanambi, BA, 2010 a 2012.

\begin{tabular}{|c|c|c|c|c|c|c|c|c|}
\hline \multirow{2}{*}{ Características } & \multirow{2}{*}{ Ciclo } & \multicolumn{7}{|c|}{ Cultivares } \\
\hline & & Maravilha & BRS Fhia-18 & Fhia-18 & BRS Platina & Prata-Anã & JV42-135 & $\mathrm{CV}(\%)$ \\
\hline \multirow{2}{*}{$\mathrm{APF}(\mathrm{cm})$} & $1^{\mathrm{o}}$ & $310,70 \mathrm{~B}$ & $249,40 \mathrm{C}$ & $303,30 \mathrm{~B}$ & $285,70 \mathrm{~B}$ & $295,10 \mathrm{~B}$ & $372,70 \mathrm{~A}$ & 4,94 \\
\hline & $2^{\circ}$ & $383,20 \mathrm{~B}$ & $317,20 \mathrm{D}$ & $385,80 \mathrm{~B}$ & $332,10 \mathrm{CD}$ & $348,70 \mathrm{C}$ & $466,80 \mathrm{~A}$ & 4,08 \\
\hline \multirow{2}{*}{ PPNS (cm) } & $1^{\circ}$ & $106,00 \mathrm{AB}$ & $86,97 \mathrm{D}$ & $107,21 \mathrm{~A}$ & $93,75 \mathrm{C}$ & $94,67 \mathrm{C}$ & $100,60 \mathrm{~B}$ & 8,51 \\
\hline & $2^{\circ}$ & $130,80 \mathrm{~A}$ & $102,90 \mathrm{C}$ & $125,80 \mathrm{AB}$ & $109,80 \mathrm{C}$ & $121,80 \mathrm{~B}$ & $117,90 \mathrm{~B}$ & 3,48 \\
\hline \multirow{2}{*}{ NFF (un) } & $1^{\mathrm{o}}$ & $16,45 \mathrm{AB}$ & $12,20 \mathrm{C}$ & $15,36 \mathrm{~B}$ & $16,25 \mathrm{AB}$ & $17,95 \mathrm{~A}$ & $16,15 \mathrm{AB}$ & 6,68 \\
\hline & $2^{\circ}$ & $16,05 \mathrm{~B}$ & $13,80 \mathrm{~B}$ & $14,18 \mathrm{~B}$ & $16,10 \mathrm{~B}$ & $18,93 \mathrm{~A}$ & $13,70 \mathrm{~B}$ & 8,66 \\
\hline \multirow{2}{*}{$\operatorname{AFT}\left(\mathrm{m}^{2}\right)$} & $1^{\circ}$ & $16,15 \mathrm{~A}$ & $9,88 \mathrm{C}$ & $15,05 \mathrm{~B}$ & $13,84 \mathrm{~B}$ & $14,86 \mathrm{~B}$ & $16,38 \mathrm{~A}$ & 9,39 \\
\hline & $2^{\circ}$ & $15,98 \mathrm{AB}$ & $12,70 \mathrm{~B}$ & $14,30 \mathrm{~B}$ & $14,19 \mathrm{~B}$ & $18,38 \mathrm{~A}$ & $15,95 \mathrm{AB}$ & 11,87 \\
\hline \multirow{2}{*}{ IAF $\left(\mathrm{m}^{2} \mathrm{~m}^{-2}\right)$} & $1^{\mathrm{o}}$ & $2,69 \mathrm{~A}$ & $1,64 \mathrm{C}$ & $2,50 \mathrm{~B}$ & $2,30 \mathrm{~B}$ & $2,47 \mathrm{~B}$ & $2,73 \mathrm{~A}$ & 9,39 \\
\hline & $2^{\circ}$ & $2,66 \mathrm{AB}$ & $2,11 \mathrm{~B}$ & $2,38 \mathrm{~B}$ & $2,36 \mathrm{~B}$ & $3,06 \mathrm{~A}$ & $2,65 \mathrm{AB}$ & 11,87 \\
\hline \multirow{2}{*}{ NFC (un) } & $1^{\mathrm{o}}$ & $11,90 \mathrm{ABC}$ & $9,70 \mathrm{D}$ & $10,13 \mathrm{CD}$ & $12,10 \mathrm{AB}$ & $13,68 \mathrm{~A}$ & $11,50 \mathrm{BCD}$ & 8,4 \\
\hline & $2^{\circ}$ & $12,80 \mathrm{~B}$ & $10,60 \mathrm{C}$ & $9,83 \mathrm{C}$ & $12,53 \mathrm{~B}$ & $15,56 \mathrm{~A}$ & $10,60 \mathrm{C}$ & 6,59 \\
\hline \multirow{2}{*}{ MPE (kg) } & $1^{\mathrm{o}}$ & $33,72 \mathrm{~A}$ & $20,86 \mathrm{BC}$ & $24,02 \mathrm{~B}$ & $21,36 \mathrm{~B}$ & $15,58 \mathrm{C}$ & $20,47 \mathrm{BC}$ & 12,04 \\
\hline & $2^{\circ}$ & $39,40 \mathrm{~A}$ & $27,34 \mathrm{~B}$ & $30,07 \mathrm{~B}$ & $21,34 \mathrm{C}$ & $19,55 \mathrm{C}$ & $19,90 \mathrm{C}$ & 11,1 \\
\hline \multirow{2}{*}{ NPE (un) } & $1^{\mathrm{o}}$ & $9,30 \mathrm{~B}$ & $9,70 \mathrm{~B}$ & $11,06 \mathrm{~A}$ & $9,20 \mathrm{~B}$ & $9,86 \mathrm{~B}$ & $7,86 \mathrm{C}$ & 3,81 \\
\hline & $2^{\circ}$ & $10,95 \mathrm{~B}$ & $10,80 \mathrm{~B}$ & $13,00 \mathrm{~A}$ & $9,60 \mathrm{C}$ & $11,51 \mathrm{~B}$ & $8,60 \mathrm{D}$ & 3,91 \\
\hline \multirow{2}{*}{ NFR (un) } & $1^{\mathrm{o}}$ & $145,60 \mathrm{~B}$ & $145,46 \mathrm{~B}$ & $181,15 \mathrm{~A}$ & $147,85 \mathrm{~B}$ & $149,18 \mathrm{~B}$ & $121,40 \mathrm{C}$ & 6,68 \\
\hline & $2^{\circ}$ & $175,00 \mathrm{BC}$ & $175,30 \mathrm{BC}$ & $225,50 \mathrm{~A}$ & $150,70 \mathrm{CD}$ & $190,50 \mathrm{~B}$ & $135,60 \mathrm{D}$ & 7,74 \\
\hline \multirow{2}{*}{$\operatorname{MFR}(g)$} & $1^{\circ}$ & $240,15 \mathrm{~A}$ & $153,16 \mathrm{BC}$ & $137,0 \mathrm{CD}$ & $156,0 \mathrm{BC}$ & $115,33 \mathrm{D}$ & $175,83 \mathrm{~B}$ & 9,3 \\
\hline & $2^{\circ}$ & $245,80 \mathrm{~A}$ & $178,00 \mathrm{~B}$ & $139,50 \mathrm{CD}$ & $154,10 \mathrm{BC}$ & $119,00 \mathrm{D}$ & $148,00 \mathrm{BCD}$ & 10,0 \\
\hline \multirow{2}{*}{$\mathrm{CFR}(\mathrm{cm})$} & $1^{\mathrm{o}}$ & $24,95 \mathrm{~A}$ & $20,53 \mathrm{BC}$ & $19,43 \mathrm{C}$ & $20,50 \mathrm{BC}$ & $17,31 \mathrm{D}$ & $22,20 \mathrm{~B}$ & 4,28 \\
\hline & $2^{\circ}$ & $24,30 \mathrm{~A}$ & $21,10 \mathrm{AB}$ & $18,50 \mathrm{~B}$ & $20,30 \mathrm{AB}$ & $19,80 \mathrm{AB}$ & $20,70 \mathrm{AB}$ & 12,18 \\
\hline \multirow{2}{*}{$\mathrm{DFR}(\mathrm{mm})$} & $1^{\mathrm{o}}$ & $39,75 \mathrm{~A}$ & $35,40 \mathrm{~B}$ & $34,13 \mathrm{~B}$ & $35,90 \mathrm{~B}$ & $34,60 \mathrm{~B}$ & $35,58 \mathrm{~B}$ & 5,34 \\
\hline & $2^{\circ}$ & $40,50 \mathrm{~A}$ & $36,10 \mathrm{BC}$ & $34,00 \mathrm{BCD}$ & $36,80 \mathrm{~B}$ & $33,10 \mathrm{CD}$ & $32,60 \mathrm{D}$ & 4,76 \\
\hline
\end{tabular}

APF: altura da planta; PPNS: perímetro do pseudocaule ao nível do solo; NFF: número de folhas funcionais no florescimento; AFT: área foliar total. IAF: índice de área foliar; NFC: número de folhas funcionais à colheita; MPE: massa das pencas; NPE: número de pencas; NFR: número de frutos; MFR: massa do fruto; CFR: comprimento do fruto; DFR: diâmetro do fruto;

Médias seguidas de letras iguais na linha, não diferem estatisticamente pelo Teste de Tukey a 5\% de probabilidade.

As folhas da 'BRS Fhia-18' a 13,68 folhas para 'Prata Anã' (Tabela 1). A 'Prata-Anã' reteve mais folhas que 'BRS Fhia-18', 'Fhia-18' e 'JV42-135'. No segundo ciclo de produção a 'Prata-Anã' reteve mais folhas à época da colheita, 'Maravilha' e 'BRS Platina' apresentaram valores intermediários, 'BRS Fhia-18', 'Fhia-18' e 'JV42-135', menos folhas. Plantas mais altas como a JV42-135 são mais suscetíveis ao efeito de vento (DONATO et al., 2006), fator de destruição foliar.

Para massa das pencas tanto no primeiro $(33,72 \mathrm{~kg})$ quanto no segundo $(39,40 \mathrm{~kg})$ ciclo de produção a 'Maravilha' alcançou a maior média (Tabela 1). Ainda no primeiro ciclo, 'BRS Platina' e 'Fhia-18' se igualaram e exibiram valores intermediários; 'Prata Anã' obteve desempenho semelhante à 'BRS Fhia-18' e 'JV42-135', que não diferiram da 'BRS Platina' e 'Fhia-18'. No segundo ciclo 'Prata-Anã', 'BRS Platina' e 'JV42-135' apresentaram as menores massa das pencas, 'Fhia-18' e 'BRS Fhia-18' expressaram valores intermediários. Ocorreram algumas variações nos valores de massa das pencas registrados no presente trabalho para algumas cultivares comparados aos de Donato et al. (2009), provavelmente decorrente das interações genótipos ambiente.

Analisando o número de pencas o hibrido 'Fhia-18' expressou as maiores médias nos dois ciclos avaliados com 11,0 no primeiro e 13,0 no segundo ciclo (Tabela 1). Enquanto o menor número de pencas foi registrado nos dois ciclos para 'JV42-135' que variou de 7,86 para 8,6 pencas entre ciclos. 'BRS Platina' mostrou desempenho intermediário no segundo ciclo nesse caráter. A cultivar com maior número de frutos e de pencas foi a 'Fhia-18', corroborando os resultados encontrados por Donato et al. (2009). O menor número de pencas expresso pela 'JV42-135' deve-se à genitora 'Prata de Java' diferente das demais, originadas da 'Prata-Anã'. 
Adicionalmente o maior porte pode também ter contribuído (DONATO et al., 2006).

A 'Fhia-18' apresentou as maiores quantidade de frutos nos dois ciclos de produção, 181,15 e 225,5 no primeiro e segundo ciclos, respectivamente (Tabela 1).

'Maravilha', 'BRS Fhia-18', 'BRS Platina' e 'PrataAnã' mostraram resultados semelhantes no primeiro ciclo. As menores médias foram exibidas nos dois ciclos pelo mesmo hibrido, a 'JV42-135'.

A massa dos frutos variou de $115,33 \mathrm{~g}$ na 'Prata Anã' para 240,15 g na 'Maravilha', no primeiro ciclo (Tabela 1). 'Fhia-18' não diferiu da 'Prata-Anã' e também foi similar à 'BRS Fhia-18' e 'BRS Platina', que não diferiram da 'JV42135 '. No segundo ciclo a variação da massa do fruto entre as cultivares foi semelhante.

O comprimento do fruto é utilizado para definir a classe, é importante na comercialização, haja vista que o preço de venda varia com a classificação da banana, apesar da dependência da exigência do mercado (FARIA et al., 2010), e constitui característica essencial também ao melhoramento de bananeiras, quando o objetivo é obter híbridos com maiores tamanhos de fruto. Houve nesse caráter oscilação de $17,31 \mathrm{~cm}$ no primeiro ciclo da genitora 'Prata-Anã' a 24,95 cm na 'Maravilha'. A 'Fhia-18' apresentou comprimento intermediário. No segundo ciclo houve diferença para comprimento do fruto apenas entre 'Maravilha' e 'Fhia-18'. 'Maravilha' superou as demais cultivares em diâmetro do fruto, com média de $39,75 \mathrm{~mm}$ no primeiro ciclo e $40,50 \mathrm{~mm}$ no segundo ciclo de produção. Os valores de diâmetro do fruto superam o mínimo exigido para o enquadramento na categoria Extra (PBMH; PIF, 2006).

\section{CONCLUSÕES}

Concluiu-se que 'Maravilha' é a mais produtiva, com maior massa das pencas, maior massa, comprimento e diâmetro do fruto; 'JV42-135' apresenta maior porte, menos pencas e frutos; 'BRS Fhia-18' é menos vigorosa; 'BRS Platina' assemelha-se mais à genitora, 'Prata-Anã'.

\section{REFERÊNCIAS BIBLIOGRÁFICAS}

AZEVEDO, V.F. de; DONATO, S.L.R.; ARANTES, A. de M.; MAIA, V.M.; SILVA, S. de O. e. Avaliação de bananeiras tipo Prata, de porte alto, no Semiárido. Ciência e Agrotecnologia, v.34, p.1372-1380, 2010.

AMORIM, E.P.; LESSA, L.S.; LEDO, C.A. da S.; AMORIM, V.B. de O.; REIS, R.V.; SANTOS-SEREJO, J.A. dos; SILVA, S. de O e. Caracterização agronômica e molecular de genótipos diplóides melhorados de bananeira. Revista Brasileira de Fruticultura, Jaboticabal, v.31, p.154-161, 2009.

DONATO, S.L.R.; ARANTES, A. de M.; SILVA, S. de O. e; CORDEIRO, Z.J.M. Comportamento fitotécnico da bananeira 'Prata-Anã' e de seus híbridos. Pesquisa Agropecuária Brasileira,v.44, p.1608-1615, 2009.
DONATO, S.L.R.; SILVA, S. de O. e; LUCCA FILHO, O.A.; LIMA, M.B.; DOMINGUES, H.; ALVES, J. da S. Comportamento de variedades e híbridos de bananeira (Musa spp.), em dois ciclos de produção no sudoeste da Bahia. Revista Brasileira de Fruticultura, v.28, p.139-144, 2006.

FARIA, H.C. de; DONATO, S.L.R.; PEREIRA, M.C.T.; SILVA, S. de O. e. Avaliação fitotecnica de bananeiras tipo Terra sob irrigação em condições semi-áridas. Ciência e Agrotecnologia, Lavras, v.34, p.830-836, 2010.

LÉDO, A.S.; SILVA JÚNIOR, J.F.; LÉDO, C.A.S.; SILVA, S.O. Avaliação de genótipos de bananeira na região do Baixo São Francisco, Sergipe. Revista Brasileira de Fruticultura, v.30, p.691-695, 2008.

LICHTEMBERG, L.A; LICHTEMBERG, P.S.F. Avanços na bananicultura brasileira. Revista Brasileira de Fruticultura, v.33, p.29-36, 2011.

MARQUES, P.R.R.; DONATO, S. L. R.; PEREIRA, M.C.T.; COELHO, E.F.; ARANTES, A.M. Características agronômicas de bananeira tipo Prata sob diferentes sistemas de irrigação. Pesquisa Agropecuária Brasileira, Brasília, v. 46, n.8, p.852-859, ago. 2011.

OLIVEIRA, T.K. de; LESSA, L.S.; SILVA, S. de O. e; OLIVEIRA, J.P. de. Características agronômicas de genótipos de bananeira em três ciclos de produção em Rio Branco, AC. Pesquisa Agropecuária Brasileira, v.43, p.1003-1010, 2008.

PBMH \& PIF. Programa Brasileiro para a Modernização da Horticultura \& Produção Integrada de Frutas. Normas de classificação de banana. São Paulo: CEAGESP, 2006. Documentos, 29.

RODRIGUES, F.E.; LIBRELON, S.S.; NIETSCHE, S.; COSTA, M.R.; PEREIRA, M.C.T. Genetic variability in clones of 'Prata Anã' banana based on phenotypical moleculars markers. Bragantia, v.71, p.182-189, 2012.

RODRIGUES, M.G.V.; DONATO, S.L.R.; RODRIGUES, F.E. Panorama del Sector bananero en el Norte de Minas Gerais, Brasil. Boletín Musalac, Costa Rica: Bioversity International, v.2, p.2-5, 2011.

SILVA, S. de O. e; PASSOS, A.R.; DONATO, S.L.R.; SALOMÃO, L.C.C.; PEREIRA, L.V.; RODRIGUES, M.G.V.; LIMA NETO, F.P.; LIMA, M.B. Avaliação de genótipos de bananeira em diferentes ambientes. Ciência e Agrotecnologia, v.24, p.737-748, 2003.

ZUCOLOTO, M.; LIMA, J.S. de S.; COELHO, R.I. Modelo matemático para estimativa da área foliar total de bananeira 'Prata-Anã'. Revista Brasileira de Fruticultura, v.30, p.1152-1154, 2008. 